\title{
Methods for the Study of Nuclear and Cytoplasmic Variation in Respiratory Activity of Neurospora crassa, and the Discovery of Three New Genes
}

\author{
By O. J. GILLIE \\ National Institute for Medical Research, Mill Hill, London, N.W.7 \\ (Accepted for publication 27 February 1970)
}

\begin{abstract}
SUMMARY
Three methods of detecting respiratory variation in Neurospora crassa have been investigated. They involved the addition of dyes (pontamine sky blue and eosin yellowish) tellurite or tetrazolium to a complete medium containing ethionine. The latter prevented conidiation and enabled the colours of the colonies to be seen clearly. These methods were used to distinguish nuclear and cytoplasmic cytochrome mutants (cyt-r, cyt-2, mi-r, mi-3, SG-3 and $S G$ ) from wild-type colonies on the basis of colour. Tetrazolium violet (2.5-diphenyl-3-(I-napthyl)-tetrazolium chloride) incorporated into modified Nagai medium with ethionine is recommended for distinguishing cytoplasmic mutants of Neurospora from wild-type. Certain other nuclear mutants were also distinguishable from wild-type in some of these tests. These were $a c-I, a c-2, a c r-3, a d-4$, leu-5, nit-I, oxD-I. Thirty-two wild-type strains in reciprocal crosses were tested on these media to find whether these tests would distinguish any nuclear or cytoplasmic variation. No new cytoplasmic variants were found. Three new nuclear genes, $B^{\mathrm{m}}$ (mauve on dye media), Tet- $R$ (ability to reduce tetrazolium) and $s u^{-}$(suppressor of $m i-3$ ) are described and have been mapped. All three loci were linked to the mating type locus on chromosome I. Tet-R was found in only certain wild-type strains of mating type $a$ (e.g. 74-OR8-I $a$ ), and Tet- $W$ (unable to reduce tetrazolium) was found in all wild-type strains of mating type $A$ (e.g. 74-OR23-I $A$ ) and certain wild-types of mating type $a ; f$, a suppressor of $m i-r$, also suppressed $S G-3$ and $S G$, but not $m i-3 ; s u^{-}$suppressed $m i-3$, but none of the other cytoplasmic mutants.
\end{abstract}

\section{INTRODUCTION}

The existence of cytoplasmic mutants of Neurospora crassa which have abnormal cytochromes has been known for almost as long as cytoplasmic petites in yeast have been known (Mitchell \& Mitchell, I952; Mitchell, Mitchell \& Tissières, 1953; Ephrussi, 1953). However, it has never been possible to obtain such mutants of Neurospora with ease, partly as a result of lack of specific tests. Nevertheless, about 14 different, independently obtained cytoplasmic mutants of $N$. crassa have been described in the literature. They have various different phenotypes and are recognized by various different criteria of cytoplasmic inheritance (see Table I). Twelve are recognized by their slow or abnormal growth, and the other two are primarily recognized by ultraviolet sensitivity and morphological abnormality. At least I I are believed to have abnormal cytochromes, which suggests that they are mitochondrial mutants.

More recent studies of cytoplasmic inheritance in Neurospora (Reich \& Luck, 1966) 


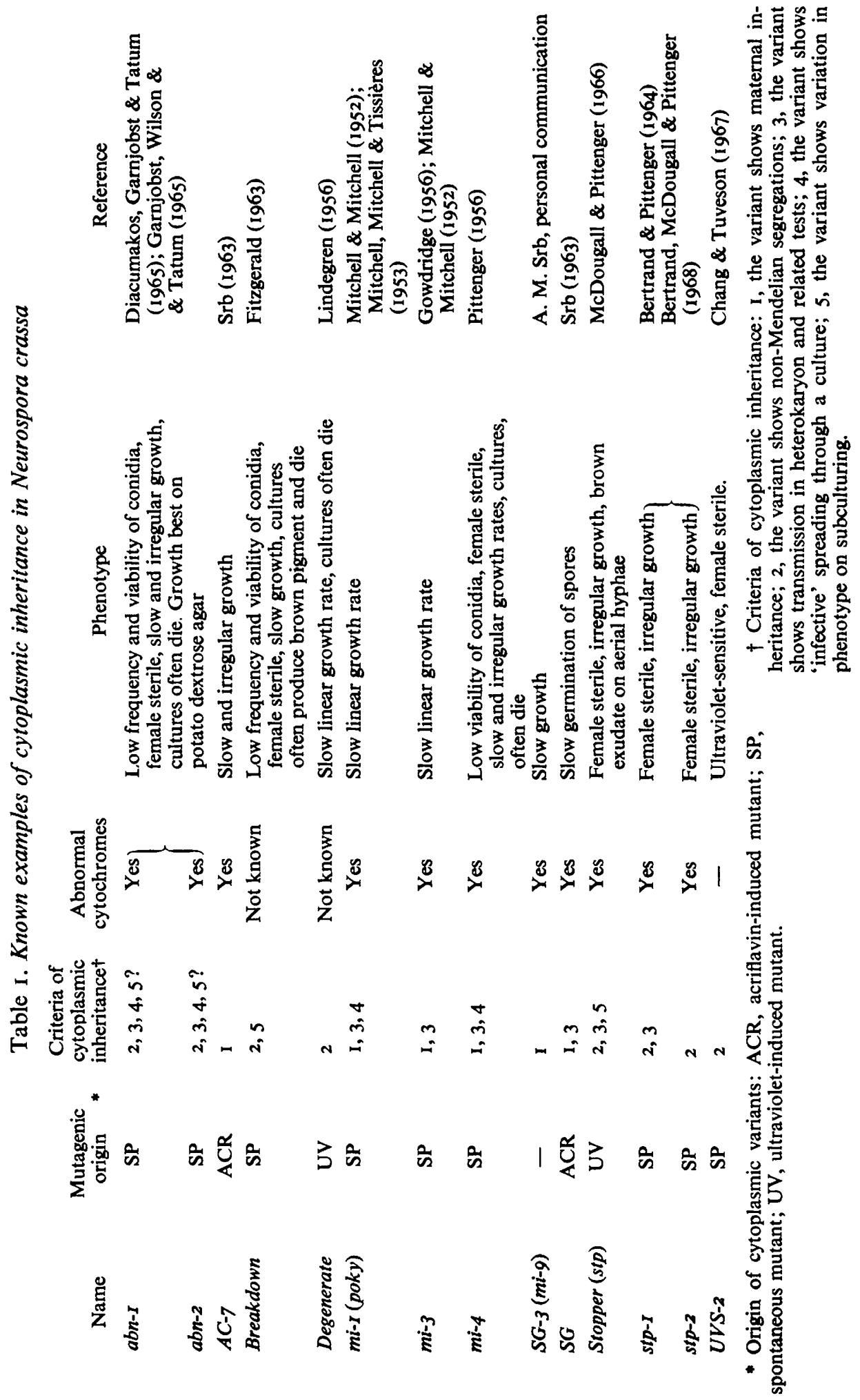


have claimed that the mitochondrial DNA as identified in caesium chloride gradients, shows maternal inheritance and that the mitochondrial structural proteins from $m i-I$ and $m i-3$ cytoplasmic mutant strains differ from the wild-type mitochondrial structural protein by one or two amino acid substitutions (Woodward \& Munkres, 1966).

This paper describes specific tests for reductive activity which have been developed in order to recognize partial defects or alterations in the mitochondrial respiratory activity of colonies of Neurospora crassa growing on plates. Although Neurospora is an obligate aerobe (Denny, 1933), variation in ability of colonies to reduce dyes, tetrazolium and sodium tellurite has been found here. These tests have been used to survey a wide selection of well-known laboratory mutants of Neurospora and also a wide selection of wild-type strains of $N$. crassa to standardize the tests and to seek further respiratory variation. Three new spontaneous nuclear mutants which affect the expression of phenotypes in these tests are described. Despite recent very impressive progress in the study of yeast mitochondrial inheritance (Thomas \& Wilkie, I968), it still seems desirable to study mitochondrial mutants in an obligate aerobe such as Neurospora which would be expected to yield mutants with more restricted, and so possibly more readily defined, defects.

\section{METHODS}

Strains. Most wild-type and mutant strains were obtained from the Fungal Genetics Stock Center, Hanover, New Hampshire, U.S.A. Information about these mutants may be obtained from Neurospora Newsletter no. I3 (I968) or from Bachmann \& Strickland (1965). The Oak Ridge wild-types 74-OR8-I $a$ and 74-OR23-I $A$ were used as standard wild-types. I am greatly indebted to Dr A. M. Srb for supplying cytoplasmic mutants $S G-3$ and $S G$ and many wild-type strains. The cytoplasmic mutants of Neurospora $m i-I$ (poky), $m i-3, S G-3$ and $S G$ were chosen for detailed investigation since they have readily recognized phenotypes. All the cytoplasmic mutants of Neurospora crassa described in the literature are listed in Table I. Of these, breakdown, degenerate, $m i-4$ and possibly $A C-7$ have been lost; $a b n-I, a b n-2, s t p, s t p-I$ and $s t p-2$ are female sterile and so the criterion of maternal inheritance cannot be used, and uvs-2 and the stp strains have a comparatively awkward phenotype to score. Cytoplasmic mutants not listed in Table I (namely, mi-2RI, mi-4RI, $m i-5 R I, m i-6 R I, m-7 R I$ and $m i-8 R I$ ) were isolated at the same time as $m i-3$ by Mitchell et al. (1953) and may be identical with $m i-3$, although $m i-8 R I$ was isolated from a different cross (Mary Mitchell, personal communication). The nuclei of all these strains, including $m i-8 R x$, were alike in containing the $B^{\mathrm{m}}$ gene described below.

A number of cytoplasmic mutant strains obtained from the Fungal Genetics Stock Center and another laboratory were found after testing to have reverted. $m i-I$ was recovered from a $[m i-r] f$ stock, which may have been prevented from further reversion by the $f$ (fast growth) gene which it already contained. $m i-3$ was recovered from a reverted stock as described below. Similar difficulties with reversion of cytoplasmic mutants have been described by Grindle \& Woodward (1968).

As suggested by Barratt (1968), cytoplasmic mutants are designated by a symbol enclosed in square brackets when it is necessary to make a distinction between nuclear and cytoplasmic mutants. 
The phenotype of the nit mutants was recognized by the production of a yellow or reddish colour on minimal slants containing $\mathrm{I} \% \mathrm{KNO}_{3}$ and $25 \mathrm{ml}$./1. bromocresol purple. $a c-I, a c-2, c y t-I, c y t-2$ and $t y-I$ were recognized by their comparatively poor growth or conidiation on minimal slants. $0 x D-I$ was identified as a methionine requirer and $o x D-8$ by its comparatively poor growth on sulphur-free minimal medium plus D-methionine (Ohnishi, Macleod \& Horowitz, 1962)

Crosses. Crosses were always made on plates of cornmeal agar at $25^{\circ}$. The female parent was inoculated first and when abundant protoperithecia had formed, after about 5 to 7 days, a suspension of conidia of the male parent was poured over the plate and left for several hours before pouring off. In describing crosses, the female or protoperithecial parent is always written first, according to the usual convention. Unless otherwise stated, plating tests always used ascospores spread in $0.0 \mathrm{I}$ to $0.5 \mathrm{ml}$. runny agar.

Media. Since anaerobically growing yeast has nutritional requirements additional to those of aerobically growing yeast (Andreasen \& Stier, 1953), it seemed desirable to use a complete medium to aid the growth of mutant strains of Neurospora crassa with partial respiratory defects. Therefore all experiments employed a complete medium modified after Nagai (1963a), which consisted of peptone $0.15 \%$, dehydrated yeast extract $0.15 \%, \mathrm{KH}_{2} \mathrm{PO}_{4} \mathrm{O} .15 \%,\left(\mathrm{NH}_{4}\right)_{2} \mathrm{SO}_{4} 0.15 \%, \mathrm{MgSO}_{4} 0.1 \%$, agar (New Zealand) $\mathrm{I} \cdot 5 \%$, sorbose $2 \%$ (added to induce colonial growth). Biotin, $5 \mathrm{mg}$. $/ \mathrm{l}$., was routinely added but can be omitted from dye media and probably also from the other media without making any difference. The $\mathrm{pH}$ of the medium was about $5 \cdot 2$. The medium was sterilized by steaming for $75 \mathrm{~min}$. in Pyrex flasks and for subsequent use re-melted by steaming for $90 \mathrm{~min}$. Before pouring plates, $2.5 \mathrm{~g}$./1. glucose and $100 \mathrm{mg}$. $/ \mathrm{l}$. DLethionine (obtained from British Drug Houses and other suppliers) were added to the medium. The ethionine was added to produce small nonconidiating colonies whose colour could be clearly seen. To prepare standard dye medium, $30 \mathrm{mg}$./1. each of pontamine sky blue (Gurr) and eosin yellowish (Gurr) were also added. For standard tellurite medium, $30 \mathrm{mg} . / 1$. $\mathrm{Na}$ tellurite were added. The glucose must be well mixed into the medium before the tellurite is added, or tellurite may be reduced directly by the glucose, turning the medium black. For tetrazolium medium, $100 \mathrm{mg} . / 1$. tetrazolium violet was added. Tetrazolium could also be used as an overlay. This consisted of $0.9 \%$ New Zealand agar containing $2.0 \mathrm{mg}$. $/ \mathrm{ml}$. red tetrazolium. In later experiments, $0.25 \mathrm{mg} . / \mathrm{ml}$. tetrazolium violet was used with better results. The overlay agar was cooled to about $45^{\circ}$ before pouring. Soda-glass bottles and Petri dishes should be avoided when using eosin yellowish pontamine sky blue media, as the dye may be decolorized in an unpredictable way on the surface of the glass. The medium was prepared in Pyrex conical flasks, and could be stored in these for weeks or possibly months. Autoclaved medium was darker than steam-sterilized medium and did not give such good colour distinction between $B^{\mathrm{m}}$ and $B^{+}$colonies. Dye solutions were kept in the refrigerator for several months without losing activity and plastic Petri plates containing the medium could be kept at room temperature for several days (so long as they were not in direct sunlight) without deteriorating. Different batches of dye have been used and no difference in the behaviour of the medium has been noted as a result of this.

Four different types of tetrazolium obtained from British Drug Houses were used: red tetrazolium, or 2,3,5-triphenyl-tetrazolium chloride; blue tetrazolium, or $3,3^{1}$ - 
diamisole-bis-4,4-(3,5-diphenyl)-tetrazolium chloride; tetrazolium violet, or 2-5diphenyl-3-(I-napthyl)-tetrazolium chloride; INP tetrazolium, or 2-(p-iodophenyl)-3(p-nitrophenyl-5-phenyl)-tetrazolium chloride.

\section{RESULTS}

\section{Media}

Dye media. Nagai (1963a, $b, 1965 a)$ found that addition of certain dyes to the medium resulted in the coloration of respiratory competent yeast colonies but not of respiratory deficient (petite) colonies. Experiments were conducted to see if dyes could be used to distinguish wild-type Neurospora from $m i-3$ and other maternally inherited mutants with abnormal cytochromes.

In preliminary experiments with dyes added to modified Nagai medium, colonies at the time of scoring were often covered with powdery conidia which prevented the true colour being observed from above, and colony colour could not be observed satisfactorily from below. However, the addition of DL-ethionine to the medium totally inhibited conidiation and enabled the true colours to be observed from above by reflected light. The 'anticonidial' effect of ethionine in liquid medium has been described by Strauss (1958).

Of a number of dyes that were tested, pontamine sky blue with eosin yellowish gave best results, staining wild-type colonies (740R8-I $a$ or 74OR23-I $A$ ) bright blue, whereas $m i-3$ colonies were pale. The mycelium of $m i-3$ colonies was stained in some places with purplish flecks of dye. The morphology also of the mi-3 colonies was abnormal compared with the wild-type; $m i-3$ colonies were very flat but were of more or less the same diameter as the wild-type.

Other dyes which distinguished between mi-3 and wild-type were aniline blue (Gurr), azorubin S (Gurr) and Magdala red (Gurr). Little or no distinction between $m i-3$ and wild-type was possible with azur $A$, malachite green, nile blue, phloxine, rose bengal and trypan blue from Gurr, and Evans blue, erythrosin B and thymol blue from British Drug Houses.

Other cytoplasmic mutants also could be distinguished from the wild-type by means of standard dye media; they all appeared thin and colourless on the first and second day, but eventually stained blue as the wild-type did. By the third day after plating, $S G$ and $[m i-I] f$ were of the same size and colour as 2-day-old wild-type colonies. $m i-3$ and $S G-3$ took 4 to 5 days to reach the same size and colour, and $m i-I$ in the absence of the $f$ (fast growth) nuclear gene took 5 or more days. mi-2RI, mi-4RI, mi-5RI, mi-6RI, $m i-7 R I$ and $m i-8 R I$ all behaved in exactly the same way as $m i-3$, with which they may be identical. The nuclear cytochrome-defective mutants cyt-I and cyt-2 gave very characteristic thin, pale colonies on standard dye media that closely resembled those of $m i-3$, although cyt-2 colonies were scarcely visible to the naked eye after two days at $32^{\circ}$.

Since Horne \& Wilkie (1966) showed that the magdala red method described by Nagai gave the same colour reaction for respiratory deficient colonies of yeast as for certain respiratory-competent auxotrophs, it was necessary to test the specificity of the method. Twenty-seven mutants tested gave blue colonies on dye media; these included I6 different auxotrophic loci and II other loci which included albino, morphological, nitrate non-utilizing and acriflavin-resistant loci. The following mutant 
stocks gave non-blue colonies, ac-r: ac-2, acr-3, ad-4, leu-5, nit-r and oxD-I. At least 20 colonies (and as many as 100 of leu- 5 and $a d-4$ ) were tested. The results showed that the non-blue phenotype was exclusively associated with the mutant type. The colour distinctions between $m i-3, B^{+}$(blue colony), and $B^{\text {m }}$ (mauve colony) could be observed at $22^{\circ}, 25^{\circ}, 32^{\circ}$, and $35^{\circ}$. Thirty-two degrees was used for all experiments unless otherwise stated.

The $\mathrm{pH}$ of the medium could be changed from 4.8 to 5.6 without affecting these colour distinctions. At higher $\mathrm{pHs} B^{\mathrm{m}}$ colonies tended to become blue, and at lower $\mathrm{pHs}$ viability of colonies was reduced. Addition of certain substances to the medium at $\mathrm{pH} 5.2$ caused blue wild-type colonies to turn pink, red or pale. Substances which had this effect included: metallic salts of $\mathrm{Zn}, \mathrm{Cu}, \mathrm{Cd}, \mathrm{Hg}$ and $\mathrm{Co}$; $\mathrm{Na}$ azide and 2,4-dinitrophenol (uncoupling agents); malonate and fluoroacetate (Krebs cycle inhibitors); cycloheximide, 5-fluorouracil, thiamine; and glucose or sucrose in a total concentration of I g./1. or more. Presumably these additions caused greater reduction of the blue dye.

Experiments with the dyes in vitro showed that they existed in a fully oxidized form and that they could be reduced to a completely colourless form by addition of sodium borohydride. They did not act as $\mathrm{pH}$ indicators in the physiological range $(\mathrm{pH} 4.2$ to 8.0). However, $\mathrm{N} / \mathrm{IONaOH}$ rapidly decolorized eosin yellowish, and pontamine sky blue was decolorized when in solution with eosin yellowish in $\mathrm{N} / \mathrm{IONaOH}$, but not when in solution alone. Eosin yellowish was precipitated by $\mathrm{N} / \mathrm{IOHCl}$, and pontamine sky blue would coprecipitate with it but did not precipitate alone. This interaction of the two dyes could be important in vivo.

Tellurite media. Nagai ( $1965 b)$ showed that respiratory deficient and respiratory competent yeasts could be distinguished by their ability to reduce sodium tellurite in the medium to metallic tellurium. Wild-type yeast colonies reduced tellurite and turned black, whereas respiratory-deficient (petite) colonies did not. When tellurite was added to modified Nagai medium at a concentration of $30 \mathrm{mg}$./l. or to Schopfer medium ' $c$ ' (Nagai, I965b), normal wild-type Neurospora colonies grown on the medium turned grey on the second day after plating, and black on the third. Two wild-type strains, $S-2$ 'bisexual' (which behaved only as $A$ mating type in my hands) and 79a, were unable to reduce tellurite but they also gave colonies which were morphologically abnormal. Nuclear genes $B^{+}, B^{\mathrm{m}}, T e t-R$ and $T e t-W$, which affect colour on dyes and tetrazolium, all gave black colonies on tellurite.

The cytoplasmic mutants and nuclear cytochrome mutants (cyt-I and cyt-2) on tellurite media gave white or pale colonies until the fifth day after plating, when $m i-3$, $S G-3$ and $S G$ colonies turned slightly grey. $m i-I,[m i-I] f$ and $c y t-I$ colonies were all still white on the fifth day. Even when compared at equivalent colony sizes (rather than ages), the cytoplasmic mutants were markedly paler than the wild-type.

Twenty-three auxotrophic and other mutants on tellurite media gave normal black colonies. However, specific supplement had to be added to $a m^{1}, l e u-2$ and tryp-3 to give the fully wild-type phenotype. nit-I gave normal-sized, paler colonies which appeared to reduce tellurite more slowly, but nit-3 gave normal black colonies. ox $D-I$ gave white colonies which became greyer, resembling wild-type, if methionine $\left(0.2 \mathrm{mg}\right.$. $/ \mathrm{ml}$.) was added to the medium but not if $\mathrm{Na}_{2} \mathrm{SO}_{3}(0.2 \mathrm{mg} . / \mathrm{ml}$.) was added to the medium. Both compounds when added to minimal medium are known to alleviate the nutritional requirement of $o x D-I$ (Ohnishi et al. 1962). oxD-8 gave normal black 
colonies, $a d-4$ and $a c-2$ rather pale colonies, and $a c-I$ minute thin pale colonies on tellurite media.

When sodium hydrogen selenite was added to modified Nagai medium at a concentration of $30 \mathrm{mg}$. $/ \mathrm{l}$., wild-type ascospores developed into pink colonies after three days at $32^{\circ}$ due to reduction of selenite, although the viability of the spores was rather reduced. The reduction of selenite by Neurospora when added to liquid medium has previously been described (Zalokar, 1953). Bismuth sulphite $(5 \mathrm{mg} . / 1$.) in modified Nagai medium was not reduced by Neurospora colonies after 3 days. although this is readily reduced to black bismuth sulphite by Candida albicans (Nickerson, 1953).

Tetrazolium media. Reduction of tetrazolium has been observed in a wide variety of micro-organisms (British Drug Houses Handbook) and has been used to detect respiratory deficiency in yeast (Ogur, St John \& Nagai, 1957). It therefore seemed, in principle, a very good test to use to detect respiratory variation in Neurospora, although there was a belief that filamentous fungi do not reduce tetrazolium(Brock, 1958).

The tetrazolium overlay method of Ogur et al. (1957) worked well when used with modified Nagai medium in the following way. Ascospores were spread on the medium and overlaid after at least $26 \mathrm{hr}$. at $32^{\circ}$ with red tetrazolium at an optimal concentration of $2 \mathrm{mg} . / \mathrm{ml}$. in $9 \%$ agar. If the colonies were overlaid before $26 \mathrm{hr}$, results were poor and variable. After overlaying it was necessary to wait at least $24 \mathrm{hr}$ before the colour developed. By this method two genes were identified in the Oak Ridge wildtypes which affect the colour produced. Tet-R readily reduces red tetrazolium, whereas Tet-W does not. The Tet-R colonies stopped growing for 2 or 3 days after overlaying, so were smaller than Tet-W colonies at the time of scoring. The genetics of these genes is described below.

Experiments were conducted to see whether other tetrazolium compounds could also be reduced by Tet-R and Tet-W strains of Neurospora when used in agar overlay. Blue tetrazolium and INP tetrazolium were reduced by both $T e t-W$ and $T e t-R$ colonies, although blue tetrazolium gave only a very weak colour that developed after several days. Tet-W colonies reduced tetrazolium violet at high concentrations $(2 \mathrm{mg} . / \mathrm{ml}$.) but not noticeably at lower concentrations at which $\mathrm{Tet}-W$ colonies readily reduced this compound and became bright violet-red. The colour of colonies given by the reduction of tetrazolium violet at $0.25 \mathrm{mg}$. $/ \mathrm{ml}$. in the overlay was much more intense than that given by the reduction of red tetrazolium at $2 \mathrm{mg} . / \mathrm{ml}$. Because the cost of using tetrazolium violet was only slightly greater, but the results very much better, this compound was adopted for routine studies. Good results could be obtained at 25 or $35^{\circ}$ as well as $32^{\circ}$ if overlaying was done when the colonies had reached the right size (at least $\mathrm{I} \cdot 5 \mathrm{~mm}$ ). At $22^{\circ}$ INP tetrazolium and tetrazolium violet worked as at other temperatures, but red and blue tetrazolium gave very poor results.

If overlaid with tetrazolium at $26 \mathrm{hr}$ the cytoplasmic mutants developed little or no colour whereas the wild-type developed excellent colour. However, wild-type colonies were larger than the mutant colonies at this age and younger, smaller wild-type colonies did not reduce tetrazolium. For this reason, colonies of cytoplasmic mutants were overlaid with tetrazolium when about the same size (at least $2 \mathrm{~mm}$. in diameter by about I mm. high) as the wild types are at $26 \mathrm{hr}$. The response of four different cytoplasmic mutants to tetrazolium overlay was tested in this way in the presence of the $T e t-R$ nuclear gene. Although all reduced the four different tetrazolium compounds, they did so to different degrees, and on the whole gave colonies paler than those of the 
wild-type. Experiments with tetrazolium violet at $32^{\circ}$ and overplating at daily intervals for 6 days (scoring after $24 \mathrm{hr}$ ) showed that $S G$ could be distinguished from wild-type only when overplated on the first day ( $26 \mathrm{hr}$ after inoculation); [mi-r] $f$ gave reddest colonies when overlaid on the fifth day after inoculation; $m i-3$ and $S G-3$ colonies never became very red, and $m i-I$ colonies were always very pale but became pink at the edges when overlaid after four days.

Since growth of some fungi is completely inhibited by quite low concentrations of tetrazolium (Brock, I958), and since tetrazolium itself can induce the formation of 'petites' in yeast (Laskowski, I954), it is usually thought necessary that tetrazolium be used as an overlay. However, tetrazolium violet or INP tetrazolium could be put into the medium itself at appropriate concentrations without preventing the growth of Neurospora colonies or markedly decreasing the viability of ascospores. This method is much simpler than the overlay method and using tetrazolium violet at $32^{\circ}$ gave excellent distinction between $T e t-R$ and $T e t-W$ colonies after 24 to $48 \mathrm{hr}$ INP tetrazolium stained both $T e t-R$ and $T e t-W$ colonies after $24 \mathrm{hr}$ Red and blue tetrazolium gave rather poor colour which did not develop well until after 5 to 6 days at any of the temperatures used.

This method was also much superior for distinguishing between cytoplasmic mutants and wild-type and was also cheaper. All the cytoplasmic mutants tested (in the presence of $T e t-R$ nuclear gene) were easily distinguishable from wild-type on tetrazolium violet medium at $32^{\circ}$ for at least 4 days after plating. By the fifth and sixth days $S G$, [mi-I] $f$, and $S G-3$ appeared much more like wild-type, but $m i-I$ and $m i-3$ still appeared lightly coloured. With INP tetrazolium the cytoplasmic mutants began to colour earlier and closely resembled the wild type by the fourth day. At $22^{\circ}$ and $25^{\circ}$ the wild-types stain most readily with INP tetrazolium, and the cytoplasmic mutants grow relatively poorly, staining very little with INP tetrazolium and not at all with tetrazolium violet. The responses at $35^{\circ}$ of wild-type and cytoplasmic mutants were the same as at $32^{\circ}$ only slightly faster.

The cytoplasmic mutants tended to grow at first as very flat colonies (whether on dye, tellurite or tetrazolium media), closely apposed to the surface of the medium. Later the edges of the colony tended to thicken and stain with the tetrazolium whether it was in the medium or in an overlay, giving the colony the appearance of a plate with a red border. Still later, the colonies of the cytoplasmic mutants thicken in the middle but always remain slightly concave, unlike the wild-type colonies which are always convex. Curiously, cyt-I colonies are raised in the centre and flat at the edges (hat-shaped), and it is the raised central portion of the colony which first becomes tetrazolium-positive.

Twenty-five auxotrophic and other mutants were tested as before to see if the mutant block interfered with reduction of tetrazolium $T e t-R$ colonies. Mutants which gave abnormal pale colonies were: $a c-1, a c-2, a d-4$ and nit-I.

\section{Genetics of $B^{\mathrm{m}}$, Tet-R and su-}

When $m i-3 A$ (2343, Fungal Genetics Stock Center no. 383) was crossed to wild-type, blue, mauve and pale colonies were obtained when it was the female parent, but only blue and mauve colonies when it was the male parent. Ten colonies of each type from each of the reciprocal crosses were picked and crossed reciprocally to wild-type, and more than 300 reciprocal crosses made amongst themselves. 
The results could be accounted for by the action of two nuclear genes, $B^{\mathrm{m}}$ which gave a mauve colony colour, and $s u^{-}$which suppressed the $m i-3$ phenotype. The pale colonies obtained when $m i-3$ was the maternal parent were the unsuppressed $m i-3$ phenotype, and the suppressed $m i-3$ phenotype appeared as blue or mauve depending on whether $B^{+}$or $B^{m}$ was present in addition to $s u^{-}$. These suppressed mi-3 colonies were indistinguishable from blue or mauve colonies with the wild-type cytoplasm. Both $B^{\mathrm{m}}$ and $s u^{-}$showed linkage to the mating type locus (Table 2) but they showed no significant linkage to each other.

\section{Table 2. Analysis of linkage of $B^{\mathrm{m}}$ and su- to mating type and to each other}

In crosses $I$ and 2 an arbitrary number of pale, blue or mauve colonies were picked and tested for mating-type. In crosses 3 to 6 the numbers of pale, blue and mauve colonies are those obtained in counts from platings of random ascospores.

\begin{tabular}{|c|c|c|c|c|c|c|}
\hline \multicolumn{7}{|c|}{ Phenotype and genotype } \\
\hline Cross & \multicolumn{2}{|c|}{ Blue } & \multicolumn{2}{|c|}{ Mauve } & \multirow{2}{*}{\multicolumn{2}{|c|}{ Pale $\left(B^{m}\right.$ or $\left.B^{+}\right)$}} \\
\hline I. $B^{+} s u^{+} a \times B^{\mathrm{m}} s u^{-} A,[m i-3]$ & $\begin{array}{c}B^{+} A \\
20\end{array}$ & $\begin{array}{c}B^{+} a \\
58\end{array}$ & $\begin{array}{c}B^{\mathrm{m}} A \\
5^{2}\end{array}$ & $\begin{array}{c}B^{\mathrm{m}} a \\
30\end{array}$ & & \\
\hline 2. $B^{\mathrm{m}} s u^{-} A,[m i-3] \times B^{+} s u^{+} a$ & $\begin{array}{c}B^{+} s u^{-} A \\
{[m i-3]} \\
27\end{array}$ & $\begin{array}{c}B^{+} s u^{-} a \\
{[m i-3]} \\
43\end{array}$ & $\begin{array}{c}B^{\mathrm{m}} s u^{-} A, \\
{[m i-3]} \\
53\end{array}$ & $\begin{array}{c}B^{\mathrm{m}} s u^{-} a \\
{[m i-3]} \\
17\end{array}$ & $\begin{array}{c}s u^{+} A \\
{[m i-3]} \\
57\end{array}$ & $\begin{array}{c}s u^{+} a \\
{[m i-3]} \\
94\end{array}$ \\
\hline $\begin{array}{l}\text { 3. } B^{\mathbf{m}} s u^{-} A,[m i-3] \times B^{+} s u^{+} A \text {, } \\
{[m i-3]}\end{array}$ & $B^{+} s u^{-}$ & {$[m i-3]$} & $B^{\mathrm{m}} s u^{-}$ & {$[m i-3]$} & $\begin{array}{l}s u^{+} \\
1087\end{array}$ & {$[m i-3]$} \\
\hline 4. $B^{+} s u^{+} a,[m i-3] \times B^{m} s u^{-} A[m i-3]$ & 34 & & & & 548 & \\
\hline $\begin{array}{l}\text { 5. } B^{\mathrm{m}} s u^{+} A,[m i-3] \times B^{+} s u^{-} a, \\
{[m i-3]}\end{array}$ & 12 & & & I & 177 & \\
\hline 6. $B^{+} s u^{-} a,[m i-3] \times B^{\mathrm{m}} s u^{+} A$, & 74 & & $6 c$ & & 1054 & \\
\hline
\end{tabular}
[mi-3]

Analysis of linkage relationships

$\begin{array}{llcc} & & & \\ & & \text { recombinants } & \text { recombination } \\ \text { Cross I } & \text { Recombination between } B \text { and } A & 50 / 160 & 31 \cdot 25 \\ \text { Cross 2 } & \text { Recombination between } B \text { and } A^{*} & 44 / 140 & 31 \cdot 4 \\ \text { Cross 2 } & \text { Recombination between } s u \text { and } A & 117 / 291 & 40 \cdot 2 \\ \text { Crosses 3 to 6 } & \text { Recombination between } s u \text { and } B & 585 / 1089,345 / 699, & 45 \text { to } 54 \dagger\end{array}$

* Calculated assuming no linkage between $\mathrm{su}^{+}$and $\mathrm{B}^{+}$, which is justified by independent measurement of this in crosses 3 to 6 , and since the same value for linkage between $B^{+}$and $A$ is obtained as in cross $I$.

$\dagger$ Test for heterogeneity of crosses 3 to 6 using the index of dispersion $\chi_{3}^{2}$ showed heterogeneity, so the results of these crosses cannot be pooled. However, the results show that recombination is between 45 and $54 \%$, ignoring cross 5 , which was rather infertile and so may have given biased results.

Amongst the 30 single colonies isolated (Io mauve, Io blue and Io pale) from the original cross in which $m i-3$ was the maternal parent, three exceptional isolates were found which in crosses to the wild-type or to other isolates did not give pale colonies and therefore could not contain the mi-3 cytoplasm. These all proved to be $s u^{+}$, and two were $B^{m}$ and one was $B^{+}$. In crosses of $s u^{+}$, [mi-3] with wild-type, occasional blue or mauve colonies were obtained when all the progeny would be expected to be pale colonies. An experiment was designed to discover whether such colonies originated from the supposed male parent forming occasional perithecia which were fertilized 
by the supposed female parent, or arose from comparatively rare transfer of male cytoplasm, or were perhaps contaminants. It seemed reasonable that comparatively rare transfer of male cytoplasm might occur since paternal inheritance of $S G$ has been described by Srb (1963).

The method used was to isolate a whole ripe perithecium and disperse its contents in I ml. of runny agar in a bijou bottle. The ascospores were activated in the bijou bottle, and then as much agar as possible was shaken out of the bottle onto a single plate, thus making a sample of the contents of a single perithecium readily identifiable. Only two wild-type blue colonies were found out of 9300 colonies examined from 234 perithecia taken from four different crosses of an $m i-3$ female crossed with a wild-type male. These two colonies came from the same perithecium, which also gave $220 \mathrm{mi}-3$ colonies. It was shown by crossing that they did not result from mutations at suppressor loci. These two colonies could have resulted from either back mutation of a mi-3 determinant or from rare transfer of determinants from the male. A control cross of an $m i-3$ female crossed with an $m i-3$ male produced 6656 pale ( $m i-3)$ colonies from 78 perithecia and no wild-type colonies. It may be concluded from these results that formation of perithecia by the 'male' parent happened rarely, if at all (no instances in 234 perithecia analysed); transfer of mi-3 male cytoplasm occurred rarely, if at all (not more than 2/9300ascospores); spontaneous back, mutation of the mi-3 determinant occurred rarely, if at all (not more than 2/15,956 ascospores); and that spontaneous mutation at suppressor loci was rare (0/15,956 ascospores). The more frequent occurrence of the wild-type colonies in the original $m i-3$ cross-analysed above may have been due to back-mutated particles having become established in the cytoplasm of the strains analysed.

Thirteen laboratory 'wild-type strains' of Neurospora (Emerson, Lindegren, St Lawrence \& Yale) were tested and all gave normal blue colonies on standard dye media except two which gave mauve colonies. It may be significant that these two strains had at one period been revived from very old subcultures kept on slants. Seventeen other wild types from different parts of the world were tested on dye media, and six of these were found to give blue colonies, five to give mauve colonies, and six to give intermediate mauvish blue colonies. These wild-types were tested in crosses with each other and with standard Oak Ridge wild-types which were made reciprocally with respect to maternal parent. No differences between reciprocal crosses (maternal inheritance) were observed.

Crosses of the two Oak Ridge wild-types (74-OR8-I $a$ and 74-OR23-I $A$ ) with each other gave both red and white colonies on tetrazolium media. In several crosses of such red and white colonies an overall proportion of 3146 red colonies to 3197 white colonies to $6 \mathrm{r}$ ambiguous or sectored colonies was obtained. The gene determining red colonies was called $T e t-R$, and its allele determining white colonies was called $T e t-W$. The locus concerned was closely linked to mating-type. Wild-type 74-OR8-I $a$ contained the $T e t-R$ gene and wild type 74-OR23-I $A$ contained $T e t-W$ gene. Of 239 progeny of a cross of $T e t-R \times T e t-W$ scored for mating type and colour on tetrazolium, 14 putative recombinants between Tet and the mating-type locus were found. The 14 putative recombinants were tested by crossing to confirm their genotypes, and four of these were found to have been misclassified or to have been assigned phenotypes which differed from their genotypes. However, of 29 non-recombinant colonies whose genotype was tested by crossing, all were correctly classified. It therefore seems justi- 
fiable to assume that misclassified colonies were of high frequency amongst putative recombinants only because misclassification would almost always have led to a colony being classified as a recombinant rather than a parental type. This experiment shows that the Tet-R locus was closely linked to the mating type locus, with only $4 \cdot 2 \%$ (10/239) recombination occurring between them.

Of the recombinants obtained, seven were $T e t-W a$ and only three Tet-RA. By means of back-crosses it was shown that these phenotypes were determined by genes allelic with the parental ones (i.e. they were not new mutants). Reciprocal crosses of all Tet-Wa recombinants with a Tet-RA tester showed that I : I segregations occurred whichever parent was the maternal parent, and therefore all the seven $T e t-W$ recombinants were genuine and mutation of a cytoplasmic particle was not involved. The small number of ambiguous or sectored colonies obtained $(6 \mathrm{I} / 6404=0.95 \%)$ occurred at rather too high a frequency to be accounted for entirely by non-disjunction of the mating-type chromosome leading to transient aneuploids (Martin, 1959) but may also have been accounted for by mixed and phenotypically variable colonies.

It was surprising that the Oak Ridge wild-types (74-OR8-I $a$ and 74-OR23-I $A$ ) differed in the presence of the $T e t-R$ or Tet-W gene, since they had been selected after extensive backcrossing in various laboratories from the original Lindegren isolates. This might have been expected to make the strains homozygous. A number of these laboratory strains of Neurospora and other wild-types from different parts of the world were analysed to see if they contained the Tet-R or Tet-W allele. The Tet-R allele was found in some of the mating type $a$, and never in the mating type $A$. All the common laboratory wild-type stocks of the $a$ mating type (Emerson $a$, Lindegren $a$, St Lawrence $a$ ) contained the Tet-R gene except for SY $4 f 8 a$ which gave white colonies. The Yale strain SY $4 f 8 a$ had been backcrossed for eight generations, and so no doubt the Tet-W gene was introduced into these stocks in the course of these crosses. Of I6 other wild types from different parts of the world, only Puerto Rico I8a gave red colonies with tetrazolium, and crosses to a Tet-RA tester strain showed that the gene involved was an allele of Tet-R. Puerto Rico $15 A$ gave white colonies in crosses with $T e t-R$, and so obeyed the rule that strains of $A$ mating type are always Tet-W.

All the other wild-types of both mating types gave only white colonies in crosses with tester strains. By making crosses reciprocal with respect to male or female parent with $T e t-R$ and $T e t-W$ tester strains, it was possible to show for 27 laboratory and other wild-types that the white or red phenotype was determined by alleles of Tet-R or Tet-W. Reciprocal crosses between wild-types' showed that there were no cytoplasmic effects on the expression of Tet-R or Tet-W. The majority of mutant strains tested of the $a$ mating type contained the Tet-R allele and the majority of mutant strains of $A$ mating type tested contained the Tet-W allele. Exceptions to this rule involved mutants on chromosome I (on which Tet and the mating-type locus are located) which presumably have been subjected to more recombination and selection involving the Tet and mating-type loci. Further investigations of the linkage relationships of Tet-R (see Table 3) were consistent with this locus being on the left arm of chromosome $\mathrm{I}$, very close to the $a d-3 B$ locus and probably located between the $a d-3 B$ and the mating-type locus. Tet-R also showed very close linkage to acr-3 (resistant to $50 \mathrm{mg}$./1. acriflavin), which may also be to the right of the mating-type locus; this arrangement is consistent with the linkage data of Hsu for the acr-3 locus (Hsu, 1965).

Mutant $s u^{-}$was found to be on the right arm of chromosome I, located between 
al-2 and nit-I (Table 3); su showed $40 \%$ recombination with Tet-R. $B^{\mathrm{m}}$ was located on the left arm of chromosome I to the left of nit-2 and showing $29.6 \%$ recombination with it (Table 3).

Table 3. Linkage data on random segregants from crosses involving the genes Tet-R, su', [mi-3] and $B^{\mathrm{m}}$.

In the data for each cross, the top number for each pair represents the progeny class which contains the top marker on the left.

\begin{tabular}{|c|c|c|c|c|c|c|}
\hline \multirow[b]{2}{*}{$\begin{array}{l}\text { Zygote genotype } \\
\text { and percentage } \\
\text { recombination }\end{array}$} & \multirow{3}{*}{$\begin{array}{c}\text { Parental } \\
\text { combina- } \\
\text { tions } \\
30\end{array}$} & \multicolumn{3}{|c|}{ Recombinations } & \multirow{3}{*}{$\begin{array}{c}\text { Total } \\
\text { isolates } \\
\text { tested } \\
73\end{array}$} & \multirow{3}{*}{$\begin{array}{l}\text { Marker } \\
\text { isolation } \\
\text { numbers } \\
\text { (Cf. first column } \\
\text { left to right) } \\
\text { m.t. }\end{array}$} \\
\hline & & $\begin{array}{l}\text { Singles } \\
\text { Region I }\end{array}$ & $\begin{array}{c}\text { Singles } \\
\text { Region II }\end{array}$ & $\begin{array}{c}\text { Doubles } \\
\text { Regions } \\
\text { I \& II }\end{array}$ & & \\
\hline$T e t-W \quad a d-3 B$ & & 3 & $\mathbf{I}$ & 0 & & \\
\hline $\begin{array}{lll}a & \text { Tet-R } & \\
8 \cdot 2 & \mathrm{I} \cdot 4\end{array}{ }^{+}$ & 36 & 3 & 0 & 0 & & $\begin{array}{l}\text { No number } \\
\text { 2-017-01 } 28\end{array}$ \\
\hline$A \quad a c r-3^{r}$ & 54 & 2 & I & 0 & 119 & m.t. \\
\hline $\begin{array}{lcc}a & a c r-3^{8} & \text { Tet }-R \\
6.7 & 1 \cdot 7\end{array}$ & 56 & 5 & 0 & $\mathbf{I}$ & & $\begin{array}{l}\text { r(KHI4) } \\
\text { No number }\end{array}$ \\
\hline$+a$ & 77 & 10 & 4 & $\mathbf{I}$ & 170 & $\mathbf{K}_{3} \mathbf{I}$ \\
\hline $\begin{array}{l}\text { Tet-W } \\
7 \cdot 1\end{array}$ & 64 & 7 & 6 & I & & $\begin{array}{l}\text { m.t. } \\
\text { No number }\end{array}$ \\
\hline$A \quad s u^{-}$ & 65 & 45 & 2 & 2 & 201 & m.t. \\
\hline $\begin{array}{ccc}a & s u^{+} & a l-2 \\
39 \cdot 8 & 4 \cdot 5 \\
& \text { o parent }[m i-3]\end{array}$ & 51 & 31 & 3 & 2 & & $\begin{array}{l}\text { No number } \\
\text { I } 5,300\end{array}$ \\
\hline$+s u^{-2}$ & 56 & 32 & 13 & 3 & 197 & m.t. \\
\hline $\begin{array}{ccc}a & \text { nit-I } & s u^{+} \\
32 \cdot 0 & I 7 \cdot 3 \\
& \text { o parent }[m i-3]\end{array}$ & $5 \mathrm{I}$ & 24 & 14 & 4 & & $\begin{array}{l}34,547 \\
\text { No number }\end{array}$ \\
\hline$B^{\mathrm{m}} \quad A$ & 12 & 12 & 16 & I I & 100 & No number \\
\hline$+{ }_{46}^{a}$ & 13 & I I & 13 & 12 & & $\begin{array}{l}\text { m.t. } \\
15,300\end{array}$ \\
\hline$+A$ & 55 & 28 & 12 & 5 & 200 & No number \\
\hline $\begin{array}{c}B^{\mathrm{m}} \\
32 \cdot 5\end{array}$ & 57 & 32 & I I & 0 & & $\begin{array}{l}\text { m.t. } \\
\text { 2-017-01 } 28\end{array}$ \\
\hline$B^{\mathbf{m}}+$ & 55 & 27 & 24 & 0 & 206 & No number \\
\hline 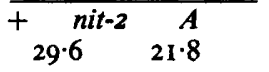 & 49 & 30 & 17 & 4 & & $\begin{array}{l}\text { K3I } \\
\text { m.t. }\end{array}$ \\
\hline
\end{tabular}

Table 4. Action of suppressors $f$ and su- on cytoplasmic mutants

Suppression of the cytoplasmic phenotype is indicated by + . No suppression is indicated by - . Suppressed cytoplasmic mutants were recognized by clearly increased colony size in crosses of the cytoplasmic mutant with $f$ or $s u^{-}$, scored after 2 or 3 days growth on Nagai medium with dyes at $32^{\circ}$.

Cytoplasmic mutant

$m i-I$

$m i-3$

$S G-3$

$S G$
Nuclear suppressor

$\begin{array}{cc}f & s u \\ + & - \\ - & + \\ + & - \\ + & -\end{array}$


A gene called $f$ (fast growth), which suppresses (or partially suppresses) the $m i-I$ phenotype, was described by Mitchell \& Mitchell (1956), and a suppressor of $m i-3$, called $s u^{-}$, is described here. Since the suppressed and unsuppressed colonies could be very clearly distinguished on dye or other indicator media, tests were made to see if either $f$ or $s u^{-}$affected cytoplasmic characters other than the one in which the suppressor was isolated. The results of these tests are given in Table 4 and show that the suppressors had different complementary actions. $s u^{-}$would only suppress $m i-3$; and $f$ suppressed $m i-I, S G-3$ and $S G$ but not $m i-3$.

\section{DISCUSSION}

Three basic methods used previously to detect respiratory deficiency in yeast have been adapted to detect respiratory variation in Neurospora crassa. The four cytoplasmic mutants $m i-1, m i-3, S G-3$ and $S G$ could be readily distinguished from wild-type on medium containing dyes, tellurite or tetrazolium. These mutants, on all three media, began with very thin growth, but could eventually form colonies almost indistinguishable in size and shape from wild-type.

All colonies of Neurospora (whether wild-type or the cytoplasmic mutant) were colourless to begin with and developed colour over a period of time on the various indicator media. The wild-type developed colour within 2 days, whereas the nuclear and cytoplasmic cytochrome mutants took longer, and on tellurite and tetrazolium media always had a paler colour than the wild-type for a given age or colony size.

All the cytoplasmic mutants examined could be distinguished on these media from the wild-type by size alone for the first 2 or 3 days, but the addition of tellurite or tetrazolium violet made it easier, particularly in the case of $S G$, which grew faster than the other cytoplasmic mutants.

Wild-type Neurospora behaved differently from wild-type yeast on dye media. Whereas wild-type yeast is colourless and respiratory-deficient yeast is purple to violet, wild-type Neurospora was blue and partially respiratory-deficient Neurospora was pale, turning blue later.

Eosin yellowish was normally reduced by Neurospora $B^{+}$colonies but not by $B^{\mathrm{m}}$ colonies. Pontamine sky blue was presumably reduced in the brilliant pink colonies produced when thiamine or additional sugar was added to the medium. It has been suggested that addition of thiamine to cultures of the fungus Rhizopus nigricans forces the anaerobic breakdown of glucose (Foster \& Goldman, 1948); this might also occur when the sugar concentration in the medium is high and might, because of changes in the organization of electron transport, cause colonies to reduce the blue dye and so become pink in colour.

None of the methods for detecting respiratory variation was entirely specific in that certain nuclear mutants which do not, as far as we know, have cytochrome abnormalities could nevertheless be recognized by their appearance on the medium. $a c-2, a d-4$ and nit-I gave large, pale-coloured colonies on all three media. This response is difficult to interpret, since a paler colour on dyes should, on the simplest interpretation, indicate a stronger reductive activity, and a paler colour on tellurite or tetrazolium a weaker reductive activity. $a c-2$ is an acetate-requiring mutant, but unfortunately little more information seems to be generally available (Perkins, Glassey \& Bloom, 1962). ad-4, an adenine-requiring auxotroph, lacks the enzyme adenylo- 
succinase and accumulates, amongst other substances, 5 -amino-4-imidazole- $N$-succinocarboxamide ribotide in the mycelium (Bernstein, 196I). This substance is colourless but becomes red on exposure to air so might well interfere with the normal reduction of the indicator substances. $a d-3 B$, which gives normal colonies on the three media, is blocked at an earlier point in the enzyme sequence and does not accumulate this substance (Bernstein, I96I). nit-I lacks nitrate reductase, but so does nit-3, which has wild-type phenotype on these media. The differences between these two nitrate nonutilizing mutants are not yet fully understood, although there seems to be a close functional relationship, if not an identity, between nitrate reductase and cytochrome $c$ reductase in Neurospora (Sorger, 1963).

Mutants acr-3 (acriflavin resistance) and leu-5 (leucine auxotroph) gave pale mauvish and pale purple colonies respectively on dye media but reduced tellurite and tetrazolium normally. $o x D-I$ gave mauve colours on dyes and did not reduce tellurite very well; this mutant lacks D-amino acid oxidase and is also unable to utilize sulphate, probably because it cannot reduce this compound (Ohnishi et al. 1962). oxD-8 also lacks D-amino acid oxidase, but is not deficient in reduction of sulphate or abnormal in its appearance on dye or tellurite media; this suggests that the abnormal phenotype of $0 \times D-I$ on these media results from its defective reduction of sulphates. $B^{\mathrm{m}}$, which was a poor reducer of dyes, reduced tellurite and tetrazolium normally, while Tet-W, which was a poor reducer of tetrazolium, reduced tellurite and dyes normally.

The most specific tests for respiratory variation were found to be those using tellurite or tetrazolium violet in the medium. These tests, particularly the latter, showed differences in colour between cytoplasmic mutants and wild-types not only at the same age but also when colonies of the same size were compared. Details of the mechanism of tellurite reduction do not appear to be known (Nagai, 1965b), but it is believed that red tetrazolium may be reduced by the action of succinic dehydrogenase (Nagai, Yanagishima \& Nagai, 196I). The ease with which tellurite and the various tetrazolium salts were reduced by Neurospora colonies did not seem to be simply related to the redox potential of the substance (redox potentials are given in Pearce, 1960) since red tetrazolium and blue tetrazolium were least easily reduced by Neurospora colonies and have redox potentials in between that of the tellurite and INP tetrazolium which were both very readily reduced by Neurospora colonies under the conditions described.

The discovery of the Tet-W-Tet-R gene difference between the supposedly isogenic Oak Ridge wild-type strains (74-OR8-I $a$ and 74-OR23-I $A$ ) is very interesting, since this genetic difference can be traced through the various laboratory wild-types and has also been discovered in the Puerto Rican wild-types. Because the real function of this gene in nature is unknown, it is impossible to speculate constructively about whether or not this genetic difference at the mating-type locus is of some mutual advantage to the two mating-types in the wild. However, the existence of this genetic difference near the mating-type locus must make us extra cautious about attributing such phenomena as poor growth of heterokaryons comprising nuclei of opposite mating-types (Gross, 1950) to the action of the mating-type locus alone. It is interesting that Hsu (I965) has discovered a spontaneous difference in acriflavin resistance between wild-type strains $74 A$ and $73 a$ (closely related to the Oak Ridge strains), due to nuclear gene $a c r-I$ closely linked to the mating type locus in chromosome $\mathrm{I}$ and therefore closely linked to Tet-R. This is further evidence that the extensive backcrossing to which the laboratory wild- 
type strains of Neurospora have been subjected (Barratt, 1962; Case, Brockman \& de Serres, 1965) have failed to make them isogenic in the region of the mating type locus. There may therefore be other gene differences closely linked to the mating-type locus which are concerned with other functions such as the actual process of mating and sexuality.

The usefulness of the media described is undoubtedly limited by the presence of sorbose to give colonial growth which must impose abnormal adjustments on the energy pathways that are indirectly involved in the processes we wish to investigate (Crocken \& Tatum, 1968). Use of colonial mutants instead of sorbose to induce colonial growth might involve similar difficulties since Brody \& Tatum (1966) have shown that one colonial mutant col-2 of Neurospora crassa has a partially defective glucose-6-phosphate dehydrogenase which must have widespread pleiotropic effects on energy metabolism. Ethionine has been shown to be incorporated into the protein of Neurospora (Kappy \& Metzenberg, 1965), but it has also been suggested that the inhibitory action of ethionine in yeast and other organisms results from its trapping of ATP (Stekol, 1963; Spence, Parks \& Shapiro, 1967). The anticonidial effect of ethionine might result from limited quantities of ATP being produced, which the organism reserves for essential growth and maintenance functions rather than for sporulation.

The observations of suppressor activity on cytoplasmic mutants first made by Mitchell \& Mitchell (1956) have been extended here to one other suppressor and two other cytoplasmic mutants. The results show that $f$ is specific in suppressing $m i-I, S G-3$ and $S G$; and $s^{-}$is specific in suppressing only $m i-3$. This shows that $m i-1, S_{3}$ and $S G$ must have related defects, although all three have readily distinguishable growth rates on plates, and $S G-3$ is most similar in colony growth rate to $m i-3$. It may be significant that Griffiths, Bertrand \& Pittenger (1968) have observed that the cytochrome spectra of $m i-I$ and $S G-3$ are similar to each other but different from wild-type and that the cytochrome spectrum of $m i-3$ differs from that of $m i-I, S G-3$ and wild-type.

During the course of the investigations described here, preliminary reports have appeared describing the differential staining of wild-type and poky colonies by means of a red tetrazolium overlay (Wilson, 1967), and 'respiratory-deficient' strains of Aspergillus nidulans have been isolated by a tetrazolium overlay method (Houghton, 1967).

\section{REFERENCES}

Andreasen, A. A. \& StiER, T. J. B. (1953). Anaerobic nutrition of Saccharomyces cerevisiae. I. Ergosterol requirement for growth in a defined medium. Journal of Cellular and Comparative Physio$\log y$ 4I, 23-36.

BachmanN, B. J. \& Strickland, W. N. (1965). Neurospora Bibliography and Index. New Haven \& London: Yale University Press.

Barratt, R. W. (1962). Origin of important wild-type stocks of $N$. crassa. Neurospora Newsletter no. 2, 24-25.

Barratt, R. W. (I968). Further notes on nomenclature: extrachromosomal mutants. Neurospora Newsletter no. 12, II.

Bertrand, H., McDougall, K. J. \& Pittenger, T. H. (I968). Somatic cell variation during uninterrupted growth of Neurospora crassa in continuous growth tubes. Journal of General Microbiology 50, 337-350.

Bertrand, H. \& Pittenger, T. H. (1964). Cytoplasmic mutants from a Neurospora culture, maintained by continuous hyphal growth. (Abstract.) Genetics 50, 235. 
BERNSTEIN, H. (196I). Imidazole compounds accumulated by purine mutants of Neurospora crassa. Journal of General Microbiology 25, 4I-46.

British Drug Houses Handbook (no date). Tetrazolium salts.

BROCK, T. D. (1958). Inhibition of growth of yeasts by 2,3,5-triphenyl tetrazolium chloride. Naturwissenschaften 45, 216.

Brody, S. \& TATUM, E. L. (1966). The primary biochemical effect of a morphological mutation in Neurospora crassa. Proceedings of the National Academy of Sciences of the United States of America 56, $1290-1297$.

Case, M. E., Brockman. H. E. \& de Serres, F. J. (1965). Further information on the origin of the Yale and Oak Ridge wild-type strains of Neurospora crassa. Neurospora Newsletter no. 8, 2526.

Chang, L. T. \& Tuveson, R. W. (1967). Ultraviolet sensitive mutants in Neurospora crassa. Genetics $56,801-810$.

Crocken, B. \& TATUM, E. L. (I968). The effect of sorbose on metabolism and morphology of Neurospora. Biochimica et Biophysica Acta 156, 1-8.

DENNY, F. E. (1933). Oxygen requirements of Neurospora sitophila for formation of perithecia and growth of mycelium. Contributions. Boyce Thompson Institute for Plant Research 5, 95-102.

Diacumakos, E. G., Garnjobst, L. \& TATuM, E. L. (1965). A cytoplasmic character in Neurospora crassa. The role of nuclei and mitochondria. Journal of Cell Biology 26, 427-443.

Ephrussi, B. (1953). Nucleo-cytoplasmic Relations in Micro-organisms: Their Bearing on Cell Heredity and Differentiation. Oxford: Clarendon Press.

FrtzGerald, P. H. (1963). Genetic and epigenetic factors controlling female sterility in Neurospora crassa. Heredity, London 18, 47-62.

Foster, J. W. \& Goldman, A. (1948). Chemical Activities of Fungi, p. 316. Edited. by J. W. Foster. New York: Academic Press.

GaRnJobst, L., WILSON, J. F. \& TATUM, E. L. (1965). Studies on a cytoplasmic character. Journal of Cell Biology 26, 412-425.

GowDRIDGE, B. (1956). Heterokaryons between strains of Neurospora crassa with different cytoplasms. Genetics 41, 780-789.

Griffiths, A. J. F., Bertrand, H. \& Pittenger, T. H. (1968). Cytochrome spectra of cytoplasmic mutants in Neurospora. Neurospora Newsletter no. 13, 16-17.

GRINDLE, M. \& WoODWARD, D. O. (1968). Variations among extrachromosomal mutants of $N$. crassa. Neurospora Newsletter no. 12, 9-10.

Gross, S. R. (1950). Heterokaryons between opposite mating types of Neurospora crassa. Biological Bulletin. Marine Biological Laboratory Woods Hole, Mass. 99 331-332.

HORNE, P. \& WILKIE, D. (1966). Use of magdala red for the detection of auxotrophic mutants of Saccharomyces cerevisiae. Journal of Bacteriology 91, 1388.

Hougrton, J. S. (1967). Respiratory deficient mutants of Aspergillus nidulans. Aspergillus Newsletter no. 8.

Hsu, K. S. (1965). Acriflavin resistance controlled by chromosomal genes in Neurospora. Neurospora Newsletter no. 8, 4-6.

KAPPY, M. S. \& METZENBERG, R. L. (1965). Studies on the basis of ethionine resistance in Neurospora. Biochimica et Biophysica Acta ro7, 425-433.

LASKOWSKI, W. (1954). Induction, par le chlorue de tetrazolium de la mutation 'petite colonie' chez la levure. Heredity, London 8, 79-88.

LiNDEGREN, C. C. (1956). Mutation and other variations in organisms. Comptes rendus des travaux du Laboratoire 26, 253-271.

MARTIN, P. G. (1959). Apparent self-fertility in Neurospora crassa. Journal of General Microbiology 20, 213.

McDougall, K. J. \& Ptrtenger, T. H. (1966). A cytoplasmic variant of Neurospora crassa. Genetics 54, 551-565.

Mrtchell, M. B. \& Mrtchell, H. K. (1952). A case of maternal inheritance in Neurospora crassa. Proceedings of the National Academy of Sciences of the United States of America 38, 442449.

Mitchell, M. B. \& MrtChell, H. K. (1956). A nuclear gene suppressor of a cytoplasmically inherited character in Neurospora crassa. Journal of General Microbiology 14, 84-89. 
MrtChell, M. B., Mitchell, H. K. \& Tissières, A. (1953). Mendelian and non-Mendelian factors affecting the cytochrome system in Neurospora crassa. Proceedings of the National Academy of Sciences of the United States of America 39, 606-6r3.

NAGAI, S. (I963a). Diagnostic colour differentiatoin plates for hereditary respiration deficiency in yeast. Journal of Bacteriology 86, 299-302.

NaGaI, S. $(1963 b)$. Aerobiosis requirement of diagnostic colour differentiation for respiration deficiency in yeast. Journal of Bacteriology 86, I I35-1 I36.

NAGAI, S. (1965a). Variety of diagnostic dye mixtures for respiration deficiency in yeast. Journal of Bacteriology 89, 897-898.

NAGA, S. $(1965 b)$. Differential reduction of tellurite by growing colonies of normal yeast and respiration deficient mutants. Journal of Bacteriology 90, 220-222.

NaGaI, S., Yanagishima, N. \& NaGaI, H. (196I). Advances in the study of respiration deficient (RD) mutation in yeast and other micro-organisms. Bacteriological Reviews 25, 404-426.

NiCKeRson, W. J. (1953). An enzymatic locus participating in cellular division of yeast. Journal of General Physiology, 37, 483-494.

OGUR, M., St John, R. \& NaGar S. (1957). Tetrazolium overlay technique for population studies of respiration deficiency in yeast. Science, New York I25, 928-929.

OHINISHi, E., MACLEOD, H. \& Horowitz, N. H. (1962). Mutants of Neurospora deficient in D-amino acid oxidase. Journal of Biological Chemistry 237, 138-142.

Pearce, A. G. E. (1960). Histochemistry-Theoretical and Applied. London: J. \&. A. Churchill.

Perkins, D. D., Glassey, M. \& Bloom, B. A. (1962). New data on markers and rearrangements in Neurospora. Canadian Journal of Genetics and Cytology 4, 187-205.

PITTENGER, T. H. (1956). Synergism of two cytoplasmically inherited mutants in Neurospora crassa. Proceedings of the National Academy of Sciences of the United States of America 42, 747-752.

ReICH, E. \& LuCK, D. J. L. (1966). Replication and inheritance of mitochondrial DNA. Proceedings of the National Academy of Sciences of the United States of America 55, 1600-1608.

SORGER, G. J. (1963). TPNH-cytochrome $c$ reductase and nitrate reductase in mutant and wild-type Neurospora and Aspergillus. Biochemical and Biophysical Research Communications 12, 395-401.

Spence, K. D., Parks, L. W. \& Shapiro, S. K. (1967). Dominant mutation for ethionine resistance in Saccharomyces cerevisiae. Journal of Bacteriology 94, I53I-I 537.

SRB, A. M. (1963). Extra-chromosomal factors in the genetic differentiation of Neurospora. Symposia of the Society for Experimental Biology 18, 175-187.

Stekol, J. A. (1963). Biochemical basis for ethionine effects on tissues. Advances in Enzymology 25, 369-393.

STRAUSS, B. S. (1958). Cell death and unbalanced growth in Neurospora. Journal of General Microbiology $18,658-669$.

ThомAS, D. Y. \& WILKIE, D. (1968). Recombination of mitochondrial drug-resistance factors in Saccharomyces cerevisiae. Biochemical and Biophysical Research Communications 30, 368-372.

WrLson, J. F. (1967). Differential effect of 2,3,5-triphenyl tetrazolium chloride on poky and wild-type colonies of Neurospora crassa. (Abstract.) Genetics 56, 597.

Woodward, D. O. \& MuNKRES, K. D. (1966). Alterations of a maternally inherited mitochondrial structural protein in respiratory deficient strains of Neurospora. Proceedings of the National Academy of Sciences of the United States of America 55, 872-880.

ZALOKAR, M. (1953). Reduction of selenite by Neurospora. Archives of Biochemistry and Biophysics 44, 330-337. 